\title{
Unanticipated rapid remission of refractory bulimia nervosa, during high-dose repetitive transcranial magnetic stimulation of the dorsomedial prefrontal cortex: a case report
}

\author{
${ }^{1}$ MRI-Guided rTMS Clinic, University Health Network, Toronto, ON, Canada \\ ${ }^{2}$ Department of Psychiatry, University of Toronto, Toronto, ON, Canada \\ ${ }^{3}$ Faculty of Medicine, University of Toronto, Toronto, ON, Canada \\ ${ }^{4}$ Program for Eating Disorders, Toronto General Hospital, Toronto, ON, Canada \\ ${ }^{5}$ Eating Disorders Program, University Health Network, Toronto, ON, Canada
}

Jonathan Downar ${ }^{1,2}{ }^{*}$, Ashwin Sankar ${ }^{3}$, Peter Giacobbe ${ }^{1,2}$, Blake Woodside $^{2,4}$ and Patricia Colton ${ }^{2,5}$

\section{Edited by:}

William McDonald, Emory University

School of Medicine, USA

\section{Reviewed by:}

Paul Croarkin, Mayo Clinic, USA

Kate Hoy, Monash University,

Australia

\section{*Correspondence:}

Jonathan Downar, Toronto Western Hospital, Room 7M-432, 399 Bathurst Street, Toronto, ON, Canada M5T $2 S 8$. e-mail: jonathan.downar@uhn.ca
A woman with severe, refractory bulimia nervosa (BN) underwent treatment for comorbid depression using repetitive transcranial magnetic stimulation (rTMS) of the dorsomedial prefrontal cortex (DMPFC) using a novel technique. Unexpectedly, she showed a rapid, dramatic remission from BN. For 5 months pre-treatment, she had reported two 5-h bingepurge episodes per day. After rTMS session 2 the episodes stopped entirely for 1 week; after session 10 there were no further recurrences. Depression scores improved more gradually to remission at session 10. Full remission from depression and binge-eating/purging episodes was sustained more than 2 months after treatment completion. In neuroimaging studies, the DMPFC is important in impulse control, and is underactive in BN. DMPFCrTMS may have enhanced the patient's ability to deploy previously acquired strategies to avoid binge-eating and purging via a reduction in her impulsivity. A larger sham-controlled trial of DMPFC-rTMS for binge-eating and purging behavior may be warranted.

Keywords: rTMS, transcranial, bulimia, anorexia, depression, dorsomedial, prefrontal, impulsivity

\section{BACKGROUND}

Eating disorders, including anorexia nervosa (AN), bulimia nervosa (BN), and binge-eating disorder (BED), have a lifetime prevalence of $\sim 5 \%$ in women (Hudson et al., 2007), and are associated with significant morbidity and mortality (Fichter et al., 2008). Eating disorders are commonly associated with affective disorders (Mangweth et al., 2003) such as major depressive disorder (MDD). Treatment options for these disorders include cognitive behavioral therapy, pharmacotherapy, or both (Brownley et al., 2007; Mitchell et al., 2007; Shapiro et al., 2007; Vocks et al., 2010). While both treatment options are moderately effective in the short term, relapse rates are high and refractory cases are common.

Repetitive transcranial magnetic stimulation (rTMS) is an emerging option in the treatment of refractory depression (Daskalakis et al., 2008; Lam et al., 2008; Schonfeldt-Lecuona et al., 2010). To date, few studies have examined the use of rTMS in patients with eating disorders, and results have been mixed using the conventional rTMS target in the dorsolateral prefrontal cortex (DLPFC; Hausmann et al., 2004; Walpoth et al., 2008; Van den Eynde et al., 2010). However, neuroimaging studies in eating disorders suggest structural and functional abnormalities of other regions, such as the anterior cingulate cortex and the neighboring dorsomedial prefrontal cortex (DMPFC; Muhlau et al., 2007; Schafer et al., 2010; Joos et al., 2011), suggesting that these could be more effective rTMS targets. Of note, there is also a converging body of evidence from lesion, stimulation, morphometric, and functional connectivity studies highlighting the DMPFC as a critical nexus of structural and functional pathology in MDD, suggesting that the DMPFC may be an effective rTMS target for MDD as well (Stefurak et al., 2003; Koenigs et al., 2008; Koenigs and Grafman, 2009; Sheline et al., 2010; Bora et al., 2012).

Here, we report a case of a woman suffering from severe, refractory $\mathrm{BN}$, and comorbid MDD. She was originally referred for MRI-guided rTMS of the DMPFC for MDD as part of an ongoing case series. However, during treatment, she serendipitously achieved rapid, complete, and sustained remission from both bulimia and MDD symptoms that persisted for more than 2 months. This case report will focus primarily on the unexpected improvement in her BN symptoms, as the effects of DMPFCrTMS in MDD across the entire case series will be the subject of a larger future publication.

\section{CASE PRESENTATION}

\section{BACKGROUND}

A 43-year-old woman, divorced with no children, on leave from her work as a pharmacist, had a longstanding history of severe and refractory AN, binge-eating/purging type. She was first hospitalized at 15 years of age with restricted eating and extreme weight loss (height, $157 \mathrm{~cm}$, weight, $31.7 \mathrm{~kg}$; BMI, 12.9) and diagnosed with AN. She began to engage in binge-eating episodes shortly thereafter, and added self-induced vomiting and some compensatory exercise at age 20. Her first episode of depression developed 
at the same age. The frequency and severity of depressive episodes, binge-eating and self-induced vomiting increased steadily over the next 20 years.

There was a marked increase in illness severity in 2007, when her husband of 8 years ended their relationship. For approximately 4 years thereafter, she engaged in one to two binge-eating episodes per day, each lasting at least $5 \mathrm{~h}$, followed immediately by purging by vomiting. By 2010, she was engaging in binge-eating and purging almost to the exclusion of all other activities including socializing, paying bills, or doing errands. She reported that she would often remain awake throughout the night to continue these behaviors, and at times would have insufficient time to shower or dress in the morning due to the duration and intensity of the episodes. Her weight gradually increased into the normal range as a consequence of the severity of her bulimic behavior, so that her diagnosis shifted to BN. As of June of 2010, she was obliged to give up her work as a pharmacist due to the severity of her illness.

There was no history of hypomania or mania, panic disorder, obsessions or compulsions, delusions or hallucinations, or selfharm behavior, either past or present. She had never engaged in any substance use aside from one to two alcoholic drinks per month. Her past medical history was remarkable for osteopenia and poor dentition secondary to purging. Although there were no family members with any formal psychiatric diagnosis, her mother and sister were reported to have symptoms of MDD, and a maternal aunt was reported to have symptoms of AN. She did have a history of paternal physical abuse in childhood.

From age 30, she had had unsuccessful trials of multiple SSRIs, trazodone, and desipramine, with add-on treatment using risperidone, quetiapine, methylphenidate, and modafinil. She had also unsuccessfully pursued several courses of intensive treatment for eating disorders, including four admissions to inpatient programs, six admissions to day hospital programs, and participation in mindfulness-based stress reduction, cognitive behavioral therapy, occupational therapy through a community outreach program, group therapy, and intensive treatment for her trauma history. In June 2010, she had attempted a day program at the Toronto General Hospital but was unable to significantly modify her eating behavior and was obliged to withdraw. In October 2010, at a weight of $47 \mathrm{~kg}$ (BMI, 18.9), she entered a 6-week inpatient treatment and was able to normalize her eating in this setting. However, she suffered a complete relapse 4 days after discharge to outpatient treatment, returning to twice-daily $5 \mathrm{~h}$ binge-eating episodes followed by purging, to the exclusion of most other activities. These episodes continued uninterrupted for the next 5 months until the beginning of her rTMS treatment.

\section{INITIAL PRESENTATION}

In April, 2011, she was referred for rTMS to address her refractory depression, rather than her disordered eating. At her intake assessment, she reported a current episode of depression beginning in 2009 , with daily symptoms of low mood, partial anhedonia, loneliness, highly impaired concentration and decision-making abilities, social withdrawal, decreased energy and motivation, and persistent thoughts of hopelessness and self-criticism about her inability to follow through with plans. She had thoughts that life was no longer worth living, but no specific suicidal intent or plan. She reported her depressive symptoms as moderately improved on a regimen of venlafaxine XR $300 \mathrm{mg}$ once daily and desvenlafaxine $50 \mathrm{mg}$ once daily, which she had been taking for the last 3 months. Her baseline pre-treatment scores were 26 on the 17 -item Hamilton Depression Rating Scale (HamD17) and 28 on the Beck Depression Inventory-II (BDI-II) self-report, suggesting a moderate to severe level of depressive symptoms.

In addition, she reported symptoms of disordered eating with twice-daily $5 \mathrm{~h}$ binges followed by purging for the last 5 months. She had a distortion of body image, describing her own appearance as "too old" and "funny-looking." Her menses had been present over the past year. At this time her weight was $50 \mathrm{~kg}$ (BMI, 20.3). She reported her current major stressors as being primarily around loneliness, separation from her husband, difficulty following through on her plans, and difficulty managing her eating and purging behavior.

\section{TREATMENT METHODS}

Following assessment, she was accepted for treatment and underwent a preliminary MRI, followed by 20 sessions of neuronavigated rTMS, one session per weekday, over 4 weeks. Neuronavigation employed the Visor 2.0 frameless stereotaxic system (Advanced Neuro Technologies, Madison, WI, USA) to position the coil at the scalp point at minimum distance from the coordinate $(x 0, y+28$, $z+45$ ) in standard space (Talairach and Tournoux, 1988), corresponding to DMPFC. Stimulation employed a MagPro R30 rTMS device connected to the fluid-cooled Cool-DB80 coil (MagVenture, Farum, Denmark), which has windings angled at $120^{\circ}$ rather than $180^{\circ}$ to achieve greater depths of stimulation.

Each treatment session involved stimulation of right then left DMPFC with 60 trains of $10 \mathrm{~Hz}$ stimulation at $120 \%$ of resting motor threshold in $5 \mathrm{~s}$ trains with a 10 -s inter-train interval, for a total of 3000 pulses to each hemisphere, as per a previously published protocol (Hadley et al., 2011). Prior to the first session, motor thresholds for flexion of the contralateral hallux were determined for each hemisphere using previously published methods (Schutter and van Honk, 2006; Hayward et al., 2007). The primary motor cortical region for the lower extremity lies on the medial wall at depths comparable to the DMPFC. Preferential stimulation of each hemisphere was achieved by orienting the coil at $90^{\circ}$ to midline, so that the direction of current flow at the coil intersection was aimed toward the hemisphere to be stimulated. As in the previous reports, this technique produced asymmetric motor activation of the contralateral lower extremity during motor threshold determination. The same approach was used for stimulation of left and right DMPFC during treatment.

\section{COURSE AND OUTCOME}

The patient tolerated the treatment well. The only adverse effects were headache and localized scalp discomfort around the site of stimulation during the first 10 sessions, and mild pre-syncopal episodes on the first and second sessions. On both occasions, she was able to recover with a brief period of rest and complete the session.

Her depression symptoms remitted over the course of treatment on both self- and clinician-rated measures, accompanied by marked and rapid improvement in daily psychosocial functioning. 
Her baseline BDI-II score, monitored daily, dropped from 28 at baseline to 7 after 11 sessions, and remained in this range thereafter. On the HamD17, monitored weekly, her score dropped from 26 at baseline to 0 by the end of treatment.

The more striking and unexpected outcome was that, after the second session of treatment, both the binge-eating and the purging behaviors stopped abruptly (Figure 1). As previously noted, the patient had been referred for rTMS for depression and there was no prior expectation of improvements in her disordered eating. For this reason, standardized measures of eating disorder severity using standard scales are not available. However, even using the coarse measure of daily binge incidence, the degree of improvement was pronounced. Using a daily log, she reported complete remission throughout the remainder of the first week of treatment. During sessions 6-10, she reported a single binge-purge episode per day. These resolved once again by the session 11 of treatment, and did not recur thereafter. Subjectively, she reported that she found herself better able to apply strategies she had learned in therapy to redirect her thoughts and behavior away from bingeeating and purging. Specifically, she reported a reduction in her sense of urgency or impulsiveness when she had the urge to binge or purge, and an enhanced ability to make other choices and follow through on planned alternative behavior. As she continued to be successful in resisting these impulses, a sense of optimism and positive self-esteem developed, and her mood then began to improve.

Full remission of both depression and disordered eating was maintained for a period of 64 days after the last session of treatment. On days 65,70 , and 71 post-treatment, she had a single episode of binge-eating and purging. She had reported the emergence of a significant psychosocial stressor (contact with her former spouse) immediately prior to this recurrence, and requested a repeat course of rTMS to avoid relapse. A second 20-session course of rTMS was initiated on day 82 post-treatment, by which time her BDI-II had increased to 20 and her HamD17 had increased to

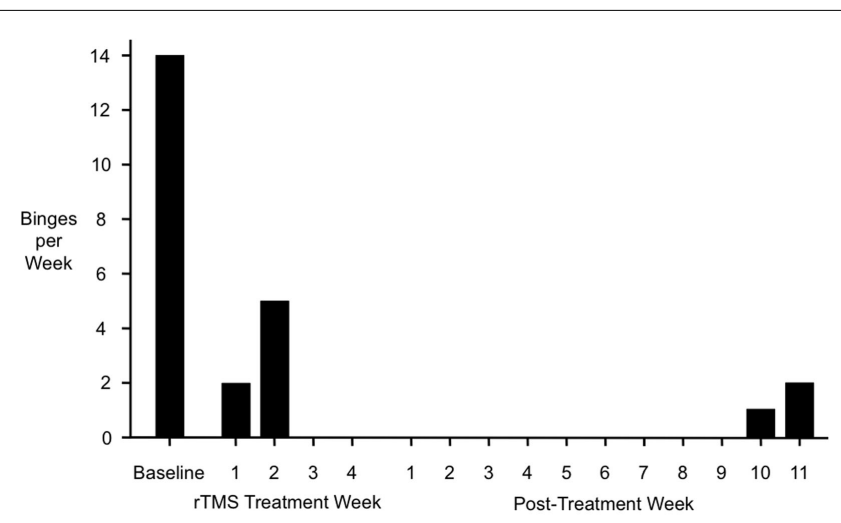

FIGURE 1 | Binge-purge episode incidence before, during, and after rTMS of the DMPFC. Full remission from binge-purge episodes was achieved by the third week of treatment, and was sustained for 9 weeks after the completion of treatment. Note that the treatment and

post-treatment periods are separated purely for schematic reasons in this figure; in terms of temporal sequence, post-treatment week 1 immediately followed rTMS treatment week 4 with no intervening period.
11. After 5 sessions of treatment these had decreased to 7 and 7 , respectively. Binge-eating and purging once again resolved completely after the first session of rTMS. She remained in remission from both depression and disordered eating throughout the rest of the course of treatment.

\section{DISCUSSION}

The presence of severe, disabling, and refractory symptoms is unfortunately very common in patients with $\mathrm{AN}$ and $\mathrm{BN}$. In this case, prior to rTMS, the patient had suffered a 20 -year history of poor response to treatment, despite the use of all available treatment modalities. Given this context, the achievement of rapid and complete remission with 20 sessions of rTMS over the DMPFC was remarkable, unanticipated, and serendipitous. The small previous literature on rTMS in disordered eating has shown mixed results with stimulation of the DLPFC. This case suggests that rTMS of the DMPFC could potentially be a more successful approach.

Although previous studies have applied short bursts of TMS to the DMPFC in non-ED patients and in healthy controls, to our knowledge this is the first report of a prolonged course of highintensity DMPFC-rTMS in eating disorders. Aside from transient local pain during the initial sessions of treatment, and mild presyncopal episodes on sessions 1 and 2, there were no adverse effects identified. Although detailed neuropsychological testing was not performed in this patient, we will note here that other patients in our clinic have undergone the same treatment protocol with either neutral or mildly beneficial effects on memory, attention, and executive function (data in preparation) - a result in keeping with previous rTMS literature on DLPFC stimulation (Guse et al., 2010).

We note that since rTMS was delivered in an open-label fashion, we cannot conclusively rule out improvement due to the non-specific effects of placebo or daily therapeutic contact. However, several factors argue against non-specific mechanisms in this case. First, the patient was undergoing treatment for depression rather than disordered eating, and neither she nor the treatment team had anticipated any effects of rTMS on her eating behavior. Second, during both courses of rTMS, the improvement in binge-eating and purging distinctly preceded the course of improvement in her symptoms of depression, rather than the other way around. The patient's subjective report was consistent with this: first, the binges abruptly resolved, then, with continuing remission, her sense of confidence and optimism began to grow and her mood improved. Third, the patient had already undergone several courses of inpatient treatment involving much more intensive, specifically directed therapies against disordered eating, with much longer periods of patient contact, yet had shown litthe sustained improvement. Fourth, in previous studies, neither active nor sham rTMS of a different target, the DLPFC, achieved improvements comparable to those in this case (Walpoth et al., 2008). Thus we consider it unlikely that any effects of expectancy, patient contact, or non-specific sensory effects of rTMS (noise, pain, etc.) are sufficient to account for the rapid, complete, and sustained remission observed in this case. Nonetheless, future studies might expand upon the present observation more rigorously by including a sham rTMS control group and by obtaining 
electrolytes and other laboratory investigations to complement the subjective reports of patients' eating diaries.

What mechanisms might account for the unanticipated success of DMPFC stimulation in this case of refractory BN? In recent years, a series of neuroimaging studies have specifically linked this brain region to impulse control and behavioral inhibition. Studies in healthy subjects have shown the DMPFC to be important in self-inhibition of actions, behaviors, and emotional reactions (Brass and Haggard, 2007; Kuhn et al., 2011). This region also activates during successful self-inhibition of loss-chasing in pathological gamblers (Campbell-Meiklejohn et al., 2008), and during food cravings (Kober et al., 2010) and cigarette cravings in smokers (Brody et al., 2007). It has also recently been shown to be hypoactive during the viewing of food cues in patients with BN (Joos et al., 2011). If the DMPFC is important for the successful inhibition of impulses to binge and purge, then rTMS-induced enhancement of its activity may explain the rapid remission of symptoms. In keeping with this proposal, a recent case report noted successful suppression of alcohol cravings in a patient with severe, refractory alcohol dependence, with rTMS of the same region (De Ridder et al., 2011).

The DMPFC is also a key region for cognitive reappraisal and the self-regulation of emotion (Wager et al., 2008). Enhancing DMPFC activity could therefore help to reduce the affective response to aversive stimuli, such as traumatic memories, or other conditioned affective responses. In this patient, the synergistic effects of improved mood, decreased anxiety, and impulsivity may have combined to normalize her eating behavior.

Another potentially important factor may have been the patient's extensive prior training in cognitive and behavioral

\section{REFERENCES}

Bora, E., Fornito, A., Pantelis, C., and Yucel, M. (2012). Gray matter abnormalities in major depressive disorder: a meta-analysis of voxel based morphometry studies. J. Affect. Disord. 138, 9-18.

Brass, M., and Haggard, P. (2007). To do or not to do: the neural signature of self-control. J. Neurosci. 27, 9141-9145.

Brody, A. L., Mandelkern, M. A., Olmstead, R. E., Jou, J., Tiongson, E., Allen, V., Scheibal, D., London, E. D., Monterosso, J. R., Tiffany, S. T., Korb, A., Gan, J. J., and Cohen, M. S. (2007). Neural substrates of resisting craving during cigarette cue exposure. Biol. Psychiatry 62, 642-651.

Brownley, K. A., Berkman, N. D., Sedway, J. A., Lohr, K. N., and Bulik, C. M. (2007). Binge eating disorder treatment: a systematic review of randomized controlled trials. Int. J. Eat. Disord. 40, 337-348.

Campbell-Meiklejohn, D. K., Woolrich, M. W., Passingham, R. E., and
Rogers, R. D. (2008). Knowing when to stop: the brain mechanisms of chasing losses. Biol. Psychiatry 63, 293-300.

Daskalakis, Z. J., Levinson, A. J., and Fitzgerald, P. B. (2008). Repetitive transcranial magnetic stimulation for major depressive disorder: a review. Can. J. Psychiatry 53, 555-566.

De Ridder, D., Vanneste, S., Kovacs, S., Sunaert, S., and Dom, G. (2011). Transient alcohol craving suppression by rTMS of dorsal anterior cingulate: an fMRI and LORETA EEG study. Neurosci. Lett. 496, 5-10.

Fichter, M. M., Quadflieg, N., and Hedlund, S. (2008). Long-term course of binge eating disorder and bulimia nervosa: relevance for nosology and diagnostic criteria. Int. J. Eat. Disord. $41,577-586$.

Guse, B., Falkai, P., and Wobrock, T. (2010). Cognitive effects of high-frequency repetitive transcranial magnetic stimulation: a systematic review. J. Neural Transm. 117, 105-122.

techniques for avoiding binge-eating and purging behaviors, acquired during previous admissions. Subjectively, she reported that she had always had difficulty consistently implementing these techniques in practice, and that an early effect of the rTMS was that she found herself more able to apply the techniques successfully. This may have reflected a decrease in her impulsivity, or in her affective response to an urge to binge or purge. Hence, the rapid response in this case could potentially reflect an enabling effect of the rTMS upon previously acquired cognitive and behavioral skills, via decreases in impulsivity and affective instability.

On a technical note, the implicated region in the neuroimaging studies of $\mathrm{BN}$ lies near the border of the anterior mid-cingulate and adjacent paracingulate cortex, at least $4 \mathrm{~cm}$ deep to scalp. Conventional flat figure- 8 coils may not be capable of inducing activation at these depths. However, the $120^{\circ}$-angled coil used in this case can generate fields of up to $0.5 \mathrm{~T}$ at $4 \mathrm{~cm}$ depth. Hence, the use of a deep-stimulating coil may be essential to the success of the treatment.

\section{CONCLUDING REMARKS}

In conclusion, this case suggests that rTMS of the DMPFC is worth further investigation as a treatment for refractory binge-eating and purging behaviors. A randomized, sham-controlled trial, in a larger group of patients, would be helpful in establishing whether the successful outcome of this case can be replicated, and to what degree non-specific mechanisms such as expectancy and therapeutic contact contribute to the overall success of the treatment. If successful, such a trial could lead to a safe, well-tolerated, and effective treatment option for patients with severe and otherwise refractory eating disorders.

Hadley, D., Anderson, B. S., Borckardt, J. J., Arana, A., Li, X., Nahas, Z., and George, M. S. (2011) Safety, tolerability, and effectiveness of high doses of adjunctive daily left prefrontal repetitive transcranial magnetic stimulation for treatment-resistant depression in a clinical setting. J. ECT 27, 18-25.

Hausmann, A., Mangweth, B., Walpoth, M., Hoertnagel, C., KramerReinstadler, K., Rupp, C. I., and Hinterhuber, H. (2004). Repetitive transcranial magnetic stimulation (rTMS) in the double-blind treatment of a depressed patient suffering from bulimia nervosa: a case report. Int. J. Neuropsychopharmacol. 7 , 371-373.

Hayward, G., Mehta, M. A., Harmer, C., Spinks, T. J., Grasby, P. M., and Goodwin, G. M. (2007). Exploring the physiological effects of double-cone coil TMS over the medial frontal cortex on the anterior cingulate cortex: an $\mathrm{H} 2(15) \mathrm{O}$ PET study. Eur. J. Neurosci. 25, 2224-2233.
Hudson, J. I., Hiripi, E., Pope, H. G. Jr., and Kessler, R. C. (2007). The prevalence and correlates of eating disorders in the National Comorbidity Survey Replication. Biol. Psychiatry 61, 348-358.

Joos, A. A., Saum, B., Zeeck, A. Perlov, E., Glauche, V., Hartmann, A., Freyer, T., Sandholz, A., Unterbrink, T., Elst, L. T., and Tuscher, O. (2011). Frontocingular dysfunction in bulimia nervosa when confronted with disease-specific stimuli. Eur. Eat. Disord. Rev. doi:10.1002/erv.1150. [Epub ahead of print].

Kober, H., Mende-Siedlecki, P., Kross, E. F., Weber, J., Mischel, W., Hart C. L., and Ochsner, K. N. (2010). Prefrontal-striatal pathway underlies cognitive regulation of craving. Proc. Natl. Acad. Sci. U.S.A. 107, 14811-14816.

Koenigs, M., and Grafman, J. (2009). The functional neuroanatomy of depression: distinct roles for ventromedial and dorsolateral prefrontal cortex. Behav. Brain Res. 201, 239-243. 
Koenigs, M., Huey, E. D., Calamia, M., Raymont, V., Tranel, D., and Grafman, J. (2008). Distinct regions of prefrontal cortex mediate resistance and vulnerability to depression. J. Neurosci. 28, 12341-12348.

Kuhn, S., Gallinat, J., and Brass, M. (2011). "Keep calm and carry on": structural correlates of expressive suppression of emotions. PLoS ONE 6, e16569. doi:10.1371/journal.pone.0016569

Lam, R. W., Chan, P., Wilkins-Ho, M., and Yatham, L. N. (2008). Repetitive transcranial magnetic stimulation for treatment-resistant depression: a systematic review and metaanalysis. Can. J. Psychiatry 53, 621-631.

Mangweth, B., Hudson, J. I., Pope, H. G., Hausmann, A., De Col, C., Laird, N. M., Beibl, W., and Tsuang, M. T. (2003). Family study of the aggregation of eating disorders and mood disorders. Psychol. Med. 33, 1319-1323.

Mitchell, J. E., Agras, S., and Wonderlich, S. (2007). Treatment of bulimia nervosa: where are we and where are we going? Int. J. Eat. Disord. 40, 95-101.

Muhlau, M., Gaser, C., Ilg, R., Conrad, B., Leibl, C., Cebulla, M. H., Backmund, H., Gerlinghoff, M., Lommer, P., Schnebel, A., Wohlschlager, A. M., Zimmer, C., and Nunnemann, S. (2007). Gray matter decrease of the anterior cingulate cortex in anorexia nervosa. Am. J. Psychiatry 164, 1850-1857.

Schafer, A., Vaitl, D., and Schienle, A. (2010). Regional grey matter volume abnormalities in bulimia nervosa and binge-eating disorder. $\mathrm{Neu}$ roimage 50, 639-643.

Schonfeldt-Lecuona, C., CardenasMorales, L., Freudenmann, R. W., Kammer, T., and Herwig, U. (2010). Transcranial magnetic stimulation in depression - lessons from the multicentre trials. Restor. Neurol. Neurosci. 28, 569-576.

Schutter, D. J., and van Honk, J. (2006). A standardized motor threshold estimation procedure for transcranial magnetic stimulation research. J. ECT 22, 176-178.

Shapiro, J. R., Berkman, N. D., Brownley, K. A., Sedway, J. A., Lohr, K. N., and Bulik, C. M. (2007). Bulimia nervosa treatment: a systematic review of randomized controlled trials. Int. J. Eat. Disord. 40, 321-336.

Sheline, Y. I., Price, J. L., Yan, Z., and Mintun, M. A. (2010). Restingstate functional MRI in depression unmasks increased connectivity between networks via the dorsal nexus. Proc. Natl. Acad. Sci. U.S.A. 107, 11020-11025.

Stefurak, T., Mikulis, D., Mayberg, H., Lang, A. E., Hevenor, S., Pahapill, P., Saint-Cyr, J., and Lozano, A. (2003). Deep brain stimulation for
Parkinson's disease dissociates mood and motor circuits: a functional MRI case study. Mov. Disord. 18, 1508-1516.

Talairach, J., and Tournoux, P. (1988). Co-Planar Stereotaxic Atlas of the Human Brain: 3-D Proportional System: An Approach to Cerebral Imaging. New York: Thieme.

Van den Eynde, F., Claudino, A. M., Mogg, A., Horrell, L., Stahl, D., Ribeiro, W., Uher, R., Campbell, I., and Schmidt, U. (2010). Repetitive transcranial magnetic stimulation reduces cue-induced food craving in bulimic disorders. Biol. Psychiatry 67, 793-795.

Vocks, S., Tuschen-Caffier, B., Pietrowsky, R., Rustenbach, S. J., Kersting, A., and Herpertz, S. (2010). Meta-analysis of the effectiveness of psychological and pharmacological treatments for binge eating disorder. Int. J. Eat. Disord. 43, 205-217.

Wager, T. D., Davidson, M. L., Hughes, B. L., Lindquist, M. A., and Ochsner, K. N. (2008). Prefrontalsubcortical pathways mediating successful emotion regulation. Neuron 59, 1037-1050.

Walpoth, M., Hoertnagl, C., MangwethMatzek, B., Kemmler, G., Hinterholzl, J., Conca, A., and Hausmann, A. (2008). Repetitive transcranial magnetic stimulation in bulimia nervosa: preliminary results of a single-centre, randomised, double-blind, sham-controlled trial in female outpatients. Psychother. Psychosom. 77, 57-60.

Conflict of Interest Statement: The authors declare that the research was conducted in the absence of any commercial or financial relationships that could be construed as a potential conflict of interest.

Received: 06 February 2012; accepted: 22 March 2012; published online: 20 April 2012.

Citation: Downar J, Sankar A, Giacobbe $P$, Woodside B and Colton P (2012) Unanticipated rapid remission of refractory bulimia nervosa, during high-dose repetitive transcranial magnetic stimulation of the dorsomedial prefrontal cortex: a case report. Front. Psychiatry 3:30. doi: 10.3389/fpsyt.2012.00030

This article was submitted to Frontiers in Neuropsychiatric Imaging and Stimulation, a specialty of Frontiers in Psychiatry. Copyright (C) 2012 Downar, Sankar, Giacobbe, Woodside and Colton. This is an open-access article distributed under the terms of the Creative Commons Attribution Non Commercial License, which permits non-commercial use, distribution, and reproduction in other forums, provided the original authors and source are credited. 